\title{
C1-C2 Instability Associated with Periodontoid Inflammatory Tissue Leading to Subarachnoid Hemorrhage: A Case Report and Review of the Literature
}

\author{
Riccardo Draghi ${ }^{1}$ Lorenzo Giammattei ${ }^{1}$ Pietro Scarone ${ }^{2}$ Giordano Lanfranchi ${ }^{1}$ Angelica Bava ${ }^{3}$ \\ Mauro Pluderi ${ }^{4}$
}

${ }^{1}$ Department of Neurosurgery, University of Milan, Milan, Italy

Address for correspondence Riccardo Draghi, MD, Neurosurgery

${ }^{2}$ Department of Neurosurgery, Lugano Regional Hospital, Lugano, Switzerland

${ }^{3}$ Istituti Clinici di Perfezionamento, Orthopaedic Trauma Center, Department, University of Milan, Via Francesco Sforza, 20122 Milan, Italy (e-mail: riccardo.draghi@gmail.com).

Rehabilitation Center for Spinal Cord Injuries, Milan, Italy

${ }^{4}$ Department of Neurosurgery, Fondazione IRCCS Ca' Granda-

Ospedale Maggiore Policlinico, Milan, Italy

Indian J Neurosurg 2015;4:180-184.

\begin{abstract}
Keywords

- periodontoid pseudotumor

- atlantoaxial instability

- subarachnoid hemorrhage

The authors present a case of atlantoaxial instability associated with C1-C2 inflammatory tissue leading to subarachnoid hemorrhage. A 65-year-old male patient arrived in June 2011 to the emergency unit for cervical pain and fever. Imaging studies documented periodontoid pseudotumor at C1-C2 level. Infective disease was suspected; the patient was therefore hospitalized and treated with antibiotics. Subsequent computed tomographic (CT) scans revealed C1-C2 instability. In August, the patient showed acute neurological deterioration and coma. Urgent brain CT revealed a hemorrhagic lesion which caused compression on the medulla oblongata, subarachnoid hemorrhage, and ventricular dilatation. An external ventricular drainage was positioned. Angio-CT and angiography did not show any vascular abnormalities. Cervical magnetic resonance imaging documented a solid tissue lesion between the atlas arch and axis. The lesion was associated with an epidural and subdural hematoma, exerting compression on brainstem. The patient underwent posterior decompression and $\mathrm{C} 1-\mathrm{C} 2$ fusion according to Harms technique in October, with significant clinical improvement. The authors present a case of atlantoaxial instability associated with a periodontoid pseudotumor at C1-C2 level determining dural sac compression. The patient showed an acute neurological deterioration caused by bleeding of the solid component of the cervical lesion. Hemorrhage of the solid component of periodontoid masses linked to atlantoaxial instability has not yet been reported in literature. To the best of our knowledge, this is the first case of $\mathrm{C} 1-\mathrm{C} 2$ instability with periodontoid pseudotumor leading to subarachnoid hemorrhage.
\end{abstract}

received

August 27, 2015

accepted

September 28, 2015

published online

December 16, 2015
DOI http://dx.doi.org/

$10.1055 / \mathrm{s}-0035-1568998$.

ISSN 2277-954X. (c) 2015 Neurological Surgeons' Society

of India
License terms

c) $(1) \risingdotseq$ 


\section{Introduction}

The authors present a singular case of atlantoaxial instability associated with $\mathrm{C} 1-\mathrm{C} 2$ inflammatory bulk tissue leading to intradural hemorrhage involving the foramen magnum and the subarachnoid space. To the best of our knowledge, this is the first case described in literature of atlantoaxial instability associated with subarachnoid bleeding.

\section{Case Report}

A 65-year-old male patient presented to the emergency department with cervical pain and fever. Standard sagittal and coronal cervical spine X-rays did not show atlantoaxial instability but underlined diffuse arthesis and inversion of the normal lordosis, thus requiring further radiological examination. Because the patient had a cardiac pacemaker, he underwent cervical spine computed tomography (CT) scan instead of magnetic resonance imaging (MRI). Imaging documented a swollen material located anteriorly to the dens at the $\mathrm{C} 1-\mathrm{C} 2$ transverse ligament level, which exerted initial dural sac compression. An infective etiology was suspected, and the patient was therefore hospitalized and treated with antibiotic therapy. Subsequent CT scans revealed $\mathrm{C} 1-\mathrm{C} 2$ instability (atlas-axis distance: $9.8 \mathrm{~mm}$ in July vs. $1.5 \mathrm{~mm}$ in May, - Fig. 1). Indication to C1-C2 posterior arthrodesis was given.

After 1 month, the patient complained of worsening cervical pain and confusion, and was again hospitalized. A few days later, gaze conjugate deviation and severe tetraparesis appeared. Urgent brain CT scan revealed a hemorrhagic hyperdensity involving the lesion, the foramen magnum, and the subarachnoid space (-Fig. 2). Compression on the medulla by the subdural hematoma and ventricular dilatation were also documented. Angio-CT and angiography excluded the presence of any vascular abnormalities. Despite the pacemaker and the risks associated, a cervical magnetic resonance imaging (MRI) was performed. The examination showed a solid hypoisointense tissue lesion located between atlas arch and axis, next to a fluid component related to a right $\mathrm{C} 1-\mathrm{C} 2$ facet joint synovial cyst. The whole lesion was associated with recent hemorrhage, which exerted compression on brainstem. The medulla and upper spinal cord showed a hyperintense signal in T2-weighted images (-Fig. 3). An external ventricular drainage (EVD) was positioned to control intracranial hypertension. Progressive improvement of the patient's clinical condition with recovery of consciousness was subsequently observed. The EVD was removed after 5 days and, 1 month after the acute clinical worsening, the patient underwent posterior decompression and C1-C2 fusion according to the Harms technique (-Fig. 4a). Biopsy of the lesion was not feasible due to the unfavorable surgical accessibility.

The patient was then transferred to a rehabilitation unit, showing progressive improvement of the neurological status. At discharge, the patient was able to walk unassisted. A cervical CT scan performed 1 year after surgery showed signs of $\mathrm{C} 1-\mathrm{C} 2$ fusion, an atlantoaxial distance of $3 \mathrm{~mm}$ and reduction of periodontoid tissue (-Fig. 4b). Brain CT documented a slight enlargement of the ventricular system.

\section{Discussion}

Atlantoaxial instability syndrome includes a wide group of pathological conditions. In fact instability can result from congenital disease, as it occurs in genomic pathologies (Morquio, Down, and Conradi syndromes) and in idiopathic dysplasias (e.g., os odontoideum, fetal warfarin syndrome) or it can be an acquired condition. In the latter group, well-known causes are infections, inflammatory

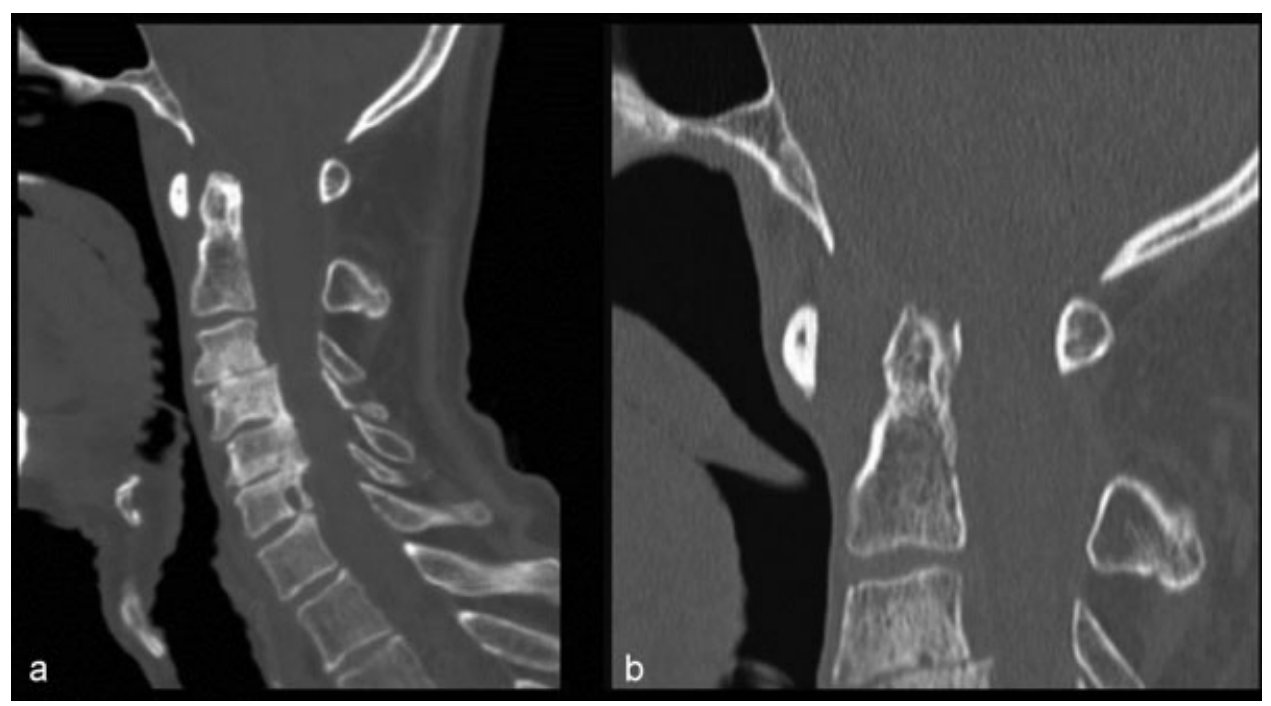

Fig. 1 (a) Sagittal CT scan (May), showing increased C1-C2 distance (1.5 mm). (b) New cervical CT (July) documented further increase of atlantoaxis distance $(9.8 \mathrm{~mm})$. CT, computed tomography. 


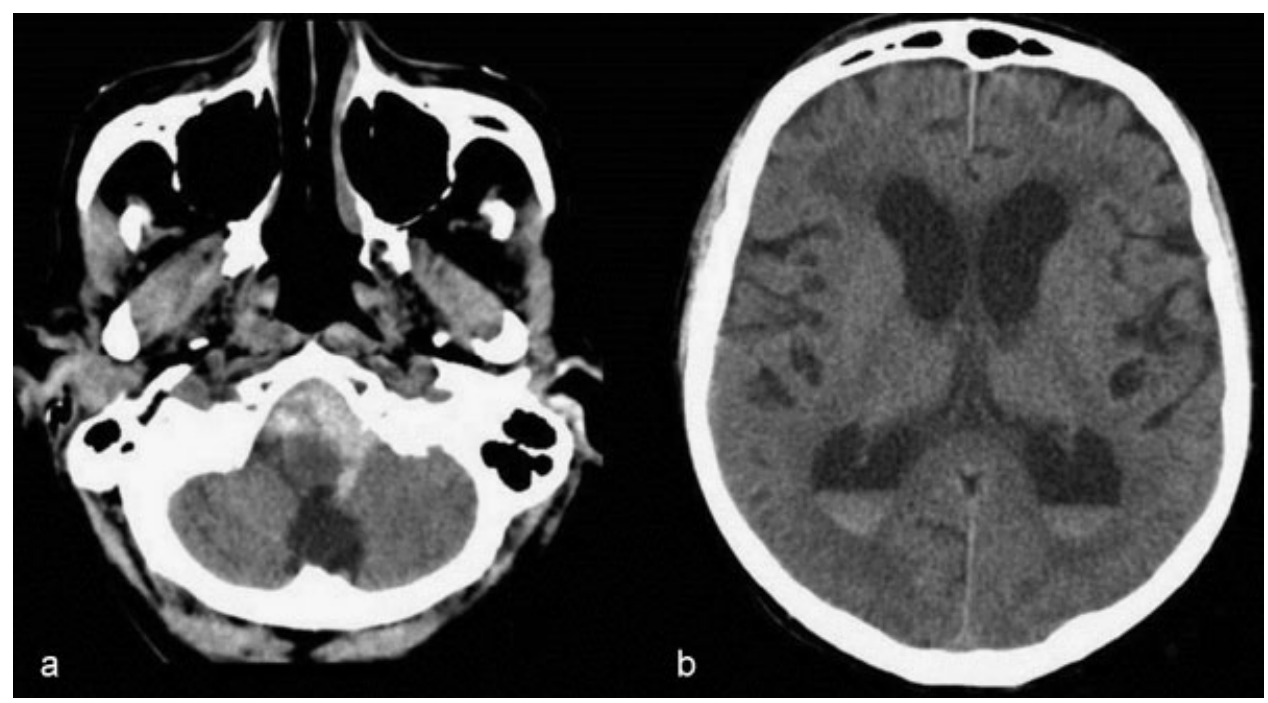

Fig. 2 (a) Axial CT demonstrates a hemorrhagic hyperdensity involving the foramen magnum and the subarachnoid space, exerting compression on the medulla oblongata. (b) Axial CT scan reveals evident ventricular dilatation; hematic hyperdensity can be seen in the occipital horns. CT, computed tomography.

diseases (in particular in rheumatoid arthritis), tumors, and traumas. $^{1}$

The development of an inflammatory mass or pannus at the craniocervical junction due to an atlantoaxial instability is a relatively common condition. ${ }^{2}$ Some authors have called these masses periodontoid pseudotumor ${ }^{3}$ or retroodontoid pseudotumor according to their position. ${ }^{4}$

However, there are some questions about the nature of this tissue. For some authors, this is an inflammatory granulation tissue, whereas for others, it is a reactive fibrous tissue due to mechanical overload. This last hypothesis would be supported by the fact that pannus is found in patients with no systemic inflammatory process and with only chronic atlantoaxial instability. ${ }^{2}$
Our clinical case represents an evident C1-C2 instability, related to a significant increase in atlantoaxial distance, as demonstrated in follow-up imaging studies ( - Fig. 1). This instability was associated with a focal mass lesion, which exerted initial compression on the dural sac, whose histological definition was not determined preoperatively because of the difficult surgical approachability.

The feature that makes this case unusual and surprising is the sudden subdural and subarachnoid hemorrhage (SAH) associated with the lesion, which caused compression of the brainstem and ventricular dilatation (-Fig. 2).

In literature, the association between $\mathrm{C} 1-\mathrm{C} 2$ instability, consequent periodontoid mass, and SAH has not been reported yet. The complete cerebral and cervical vessel

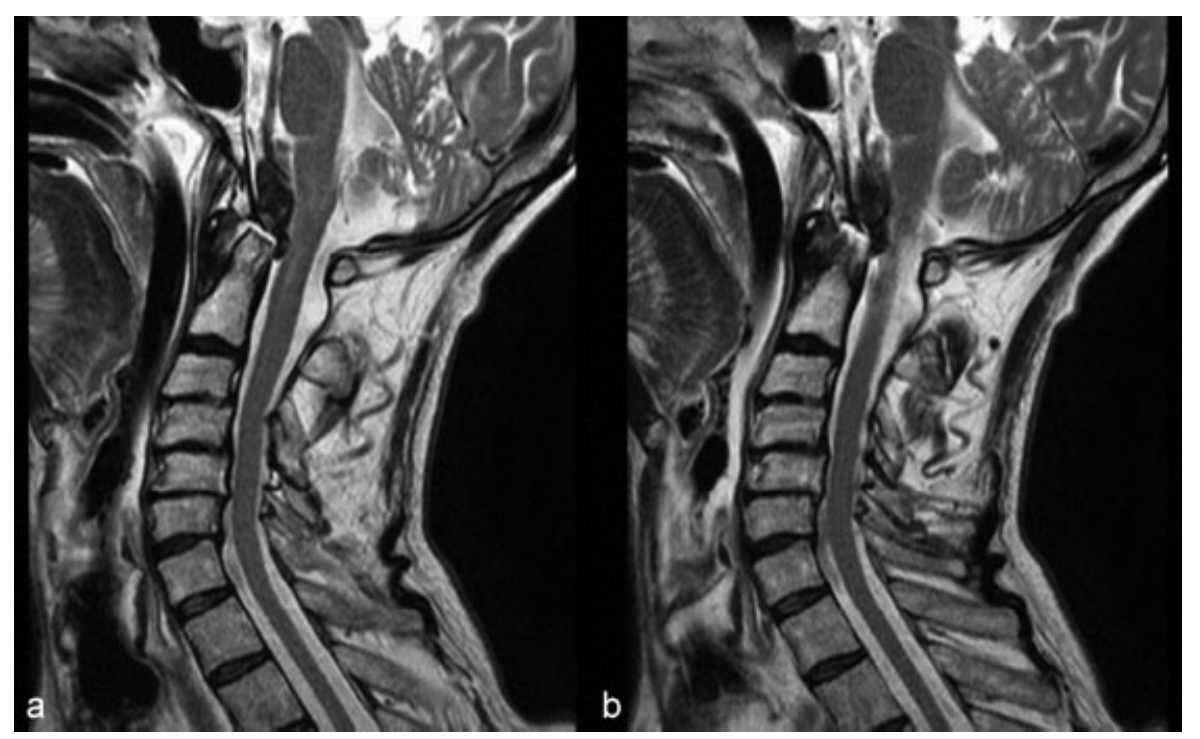

Fig. 3 (a, b) Sagittal T2-weighted MRI documents hemorrhage at C1-C2 level associated to the periodontoid lesion. The hemorrhage exerts compression on brainstem. The medulla oblongata and upper spinal tract showed a hyperintense signal. MRI, magnetic resonance imaging. 


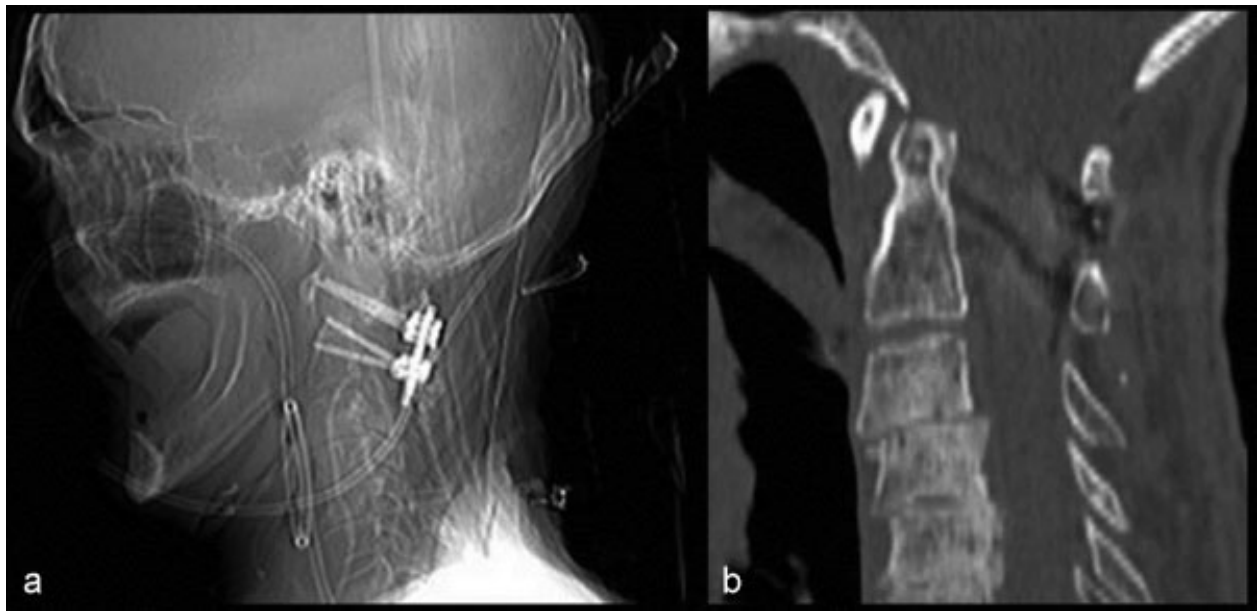

Fig. 4 (a) Lateral cervical radiogram documents posterior C1-C2 arthrodesis, according to the Harms technique. (b) Cervical CT scan performed 1 year after instrumentation demonstrates stable reduction of C1-C2 distance. CT, computed tomography.

angiography excluded the presence of any vascular abnormality and this led to the conclusion that the bleeding originated from the cervical lesion.

Radiological studies, in particular the MRI, documented a probable reactive/flogistic disease at $\mathrm{C} 1-\mathrm{C} 2$ level. The periodontoid tissue was associated with a fluid component at $\mathrm{C} 1-\mathrm{C} 2$ right joints, probably due to a synovial cyst. The presence of a synovial cyst is sign of joint instability, as Jost et al suggested. ${ }^{5}$ Even though the pathogenesis of synovial cysts is unclear, Jost et al proposed the release of inflammatory factors by stressed joints as a possible pathological mechanism, resulting in the formation of the cyst or extrusion of synovium through the joint capsule caused by trauma or instability. ${ }^{5}$ In literature, cases of hemorrhagic synovial cysts have been reported, ${ }^{6}$ but in the case presented, the bleeding involved the solid component of the lesion, as MRI clearly demonstrated ( $\mathbf{- F i g . ~ 5 ) . ~ T h e ~ s y n o v i a l ~ c y s t ~ c a n ~}$ therefore be considered an epiphenomenon of the joint instability and not the origin of the hemorrhage.

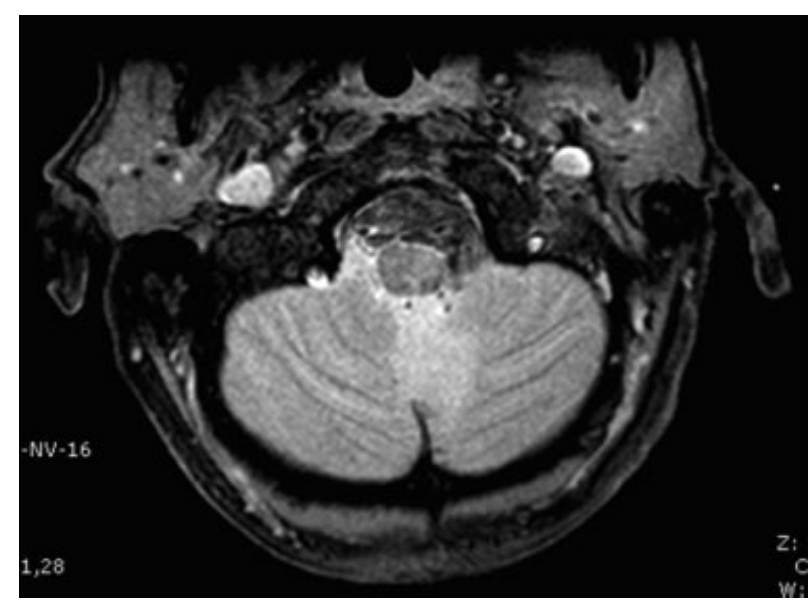

Fig. 5 Axial T2-weighted MRI showing hemorrhage of the solid component of periodontoid lesion. MRI, magnetic resonance imaging.
Hemorrhages of solid component of periodontoid masses linked to atlantoaxial instability have not, to our knowledge, been reported in the literature so far.

The hematoma determined compression of the brainstem and diffuse $\mathrm{SAH}$, with acute and massive dilatation of the ventricles, which required urgent EVD positioning. The hydrocephalus resolved during the following hospitalization, and so the shunt was removed.

In our case, there was no abnormal flourodeoxyglucose uptake noted on positron emission tomography scan and serum tumor markers were also negative. The radiological features of the lesion and the progressive craniocervical instability suggested an inflammatory disease, such as rheumatoid arthritis; however, this hypothesis was not confirmed by the analysis of autoantibodies, markers of common rheumatological diseases.

The histological diagnosis was not achieved since the lesion could not be safely reached through the posterior approach. Cases of atlantoaxial instability with periodontoid mass unrelated to rheumatoid arthritis have been reported in literature, and histological examinations in these cases showed inflammatory or scar tissue, suggesting that atlantoaxial instability induced local inflammation and then the developing of a mass. ${ }^{7}$

The mechanism, which correlated atlantoaxial instability, hemorrhage, and intradural blood diffusion, is not clear. A hypothesis could be that the atlantoaxial instability determined inflammation and frailty of the dura mater, eventually causing it to tear when the lesion bled. This mechanism would also explain blood also diffused in the subarachnoid space.

Surgical treatment of periodontoid pseudotumor can be transoral odontoid resection followed by anterior plating or, more commonly, posterior decompression and fusion. Anterior approach is recommended when atlantoaxial kyphosis is not reducible $^{8}$ and could be the best way to control ventral spinal cord compression. ${ }^{9}$ The posterior approach aims to pannus regression, due to atlantoaxial arthrodesis. $2,8,10,11$ Several reports described not only regression of periodontoid 
pseudotumor after vertebral stabilization but also clinical improvement of myelopathy. ${ }^{12}$ Pseudotumor resection allows histopathological examination but has a high risk of severe neurological deficits. In the case presented, a posterior approach with decompression and $\mathrm{C} 1-\mathrm{C} 2$ fusion with instrumentation was performed and led to progressive neurological improvement. After 1 year, the patient was able to walk again. CT cervical scan documented reduction of the periodontoid mass and of the atlas-axis distance.

\section{Conclusions}

The growth of an inflammatory mass or pannus in the craniocervical junction due to atlantoaxial instability is a well-known condition called periodontoid pseudotumor. Even if such a dramatic clinical course is exceptional a significant increase of C1-C2 distance in short-term radiological follow-up should be considered alarming and should induce an expeditious surgical treatment. In literature, there have been no cases of pannus acute bleeding leading to brainstem compression and, in particular, this could be the first case of C1-C2 instability associated with SAH.

\section{Conflict of Interest}

The authors do not declare any conflict of interest about the development and management of this case and this article.

\section{Permission Request}

The patient, even if no data can be directly linked to him/ her, declared his/her permission to publish this case report.

\section{Statement of Authors' Approval}

All the authors approve the content of the article submitted.

\section{Note}

This article was presented at the 35th National Congress of Italian Spinal Surgery Society; May 2012; Rome and at the 61st Italian Neurosurgical Society Congress; October 2012; Rimini.

\section{References}

1 Fessler RG, Sekhar L. Pathology of the occipitocervical junction. In: Dille B ed. Atlas of Neurosurgical Technique-Spine and Peripheral Nerves. New York, NY: Thieme Medical Publishers, Inc.; 1987:2-59

2 Lagares A, Arrese I, Pascual B, Gòmez PA, Ramos A, Lobato RD. Pannus resolution after occipitocervical fusion in a nonrheumatoid atlanto-axial instability. Eur Spine J 2006;15(3): 366-369

3 Sze G, Brant-Zawadzki MN, Wilson CR, Norman D, Newton TH. Pseudotumor of the craniovertebral junction associated with chronic subluxation: MR imaging studies. Radiology 1986; 161(2):391-394

4 Goel A, Phalke U, Cacciola F, Muzumdar D. Atlantoaxial instability and retroodontoid mass-two case reports. Neurol Med Chir (Tokyo) 2004;44(11):603-606

5 Jost SC, Hsien Tu P, Wright NM. Symptomatic intraosseous synovial cyst in the cervical spine: a case report. Spine 2003; 28(17):E344-E346

6 Xu R, Solakoglu C, Maleki Z, McGirt MJ, Gokaslan ZL, Bydon A. Hemorrhagic synovial cyst: the possible role of initial trauma and subsequent microtrauma in its pathogenesis: case report. Neurosurgery 2011;68(3):E858-E865, discussion E865

7 Ito K, Sakai K, Yako T, Tanaka Y, Hongo K. Atlantoaxial dislocation associated with a mass in the extradural craniovertebral junction unrelated to rheumatoid arthritis. Case report. Neurol Med Chir (Tokyo) 2007;47(4):182-185

8 Kandziora F, Mittlmeier T, Kerschbaumer F. Stage-related surgery for cervical spine instability in rheumatoid arthritis. Eur Spine J 1999;8(5):371-381

9 Moskovich R, Crockard HA. Posttraumatic atlanto-axial subluxation and myelopathy. Efficacy of anterior decompression. Spine 1990;15(6):442-447

10 Larsson EM, Holtås S, Zygmunt S. Pre- and postoperative MR imaging of the craniocervical junction in rheumatoid arthritis. AJR Am J Roentgenol 1989;152(3):561-566

11 Yamaguchi I, Shibuya S, Arima N, Oka S, Kanda Y, Yamamoto T. Remarkable reduction or disappearance of retroodontoid pseudotumors after occipitocervical fusion. Report of three cases. J Neurosurg Spine 2006;5(2):156-160

12 Chang H, Park J-B, Kim K-W, Choi WS. Retro-dental reactive lesions related to development of myelopathy in patients with atlantoaxial instability secondary to Os odontoideum. Spine 2000;25(21):2777-2783 